\title{
Role of radiation treatment in multimodality treatment of malignant pleural mesothelioma: a narrative review
}

\author{
Gillian Saldanha ${ }^{1}$, Sameera Kumar ${ }^{2}$ \\ ${ }^{1}$ Lewis Katz School of Medicine at Temple University, Philadelphia, PA, USA; ${ }^{2}$ Fox Chase Cancer Center, Department of Radiation Oncology, \\ Philadelphia, PA, USA \\ Contributions: (I) Conception and design: Both authors; (II) Administrative support: Both authors; (III) Provision of study materials or patients: \\ Both authors; (IV) Collection and assembly of data: Both authors; (V) Data analysis and interpretation: Both authors; (VI) Manuscript writing: Both \\ authors; (VII) Final approval of manuscript: Both authors. \\ Correspondence to: Sameera Kumar, MD. Department of Radiation Oncology, Fox Chase Cancer Center, 333 Cottman Avenue, Philadelphia, PA \\ 19111, USA. Email: Sameera.kumar@fccc.edu.
}

Objective: By reviewing the literature on post-operative radiotherapy, pre-operative therapy, radiotherapy for procedure-site metastases, and radiotherapy for non-operative management of malignant pleural mesothelioma (MPM), we hope to provide guidance for the role of radiotherapy in the treatment of this cancer.

Background: MPM is an aggressive rare cancer associated with high rates of morbidity and mortality. Trimodality treatment consisting of surgery, chemotherapy, and radiotherapy has shown promise for mesothelioma, as these tumors have been demonstrated to be radiosensitive.

Methods: Works of interest were identified using MEDLINE/PubMed and Cochrane Library searches. Eligible studies included those done on patients who received curative intent therapy for histologically confirmed mesothelioma.

Conclusions: Post-operative radiotherapy following either extrapleural pneumonectomy or pleurectomydecortication has shown to reduce local recurrence with increased median survival. There is limited but promising results for pre-operative radiotherapy; further research is warranted. There does not appear to be a role for prophylactic radiotherapy in procedure-site metastases. Radiotherapy may provide benefit for nonoperative management in patients that are poor surgical candidates. Based on the current body of literature, radiotherapy is especially well-suited for those with epithelioid tumors. Special attention must be paid to reduce mean lung dosages and the volume of lung receiving high radiation doses in order to lower the risk of adverse effects and pulmonary toxicity.

Keywords: Radiotherapy; malignant pleural mesothelioma (MPM); radiation therapy

Received: 06 May 2021; Accepted: 22 June 2021; Published: 30 December 2021.

doi: 10.21037/asj-21-26

View this article at: https://dx.doi.org/10.21037/asj-21-26

\section{Introduction}

Malignant pleural mesothelioma (MPM) is a rare cancer with the majority of cases resulting from occupational or environmental exposure (1). MPM is an aggressive malignancy with high rates of morbidity and mortality, with a median survival from diagnosis reported anywhere from 6 to 14 months $(2,3)$. Curative-intent therapy includes surgery as the cornerstone for MPM. This includes both extrapleural pneumonectomy (EPP) and pleurectomydecortication (P/D) as options. Trimodality therapy consisting of chemotherapy, surgery, and radiotherapy has been considered a promising approach. Studies have 
indicated that MPMs are radiosensitive tumors (4). Local relapse rates of MPM have been shown to be high in the absence of radiation therapy, with median survival rates of patients treated with surgery alone reported as low as 10 months (5).

Subtypes of mesothelioma include epithelioid, sarcomatoid, and biphasic. Typically, curative intent therapy is reserved for epithelioid and biphasic tumors, however, small-volume sarcomatoid MPM can also be considered for curative intent therapy. Radiotherapy can be used postoperatively after both EPP and P/D. Pre-operative and definitive radiotherapy are only very rarely used and not well studied (6). Three-dimensional techniques have been used in the treatment of MPM for several years, but in more recent years, intensity modulated radiation therapy (IMRT) has been shown to improve the target volume coverage with the downside of delivering greater radiation doses to the contralateral lung compared to conventional techniques (7). While an initial study of IMRT showed a substantial rate of fatal pneumonitis of almost $50 \%$ (8), recent strides in IMRT have greatly improved safety profiles (9). Volumetric arc therapy (VMAT) is a type of IMRT that dynamically delivers radiation as the gantry rotates around the patient, allowing for better dosage delivery to the tumor while sparing healthy surrounding tissues (10). We provide here a general overview of the utility, efficacy, and safety of curative intent radiation therapy for MPM. In this review we discuss topics including post-operative radiotherapy following EPP, post-operative radiotherapy following $\mathrm{P} / \mathrm{D}$, pre-operative radiotherapy, role of radiotherapy in procedure site metastases, palliative radiotherapy for nonresectable tumors, and the implications of tumor heterogeneity. We present the following article in accordance with the Narrative Review reporting checklist (available at https://asj.amegroups.com/article/ view/10.21037/asj-21-26/rc).

\section{Methods}

To identify relevant works of interest, we conducted MEDLINE/PubMed and Cochrane databases searches in February to April of 2021. The search included the following keywords: "mesothelioma AND radiotherapy" and "mesothelioma AND radiation" and was limited to clinical trials, meta-analysis, and randomized clinical trials conducted on humans. Eligible studies included those in patients with histologically confirmed mesothelioma who received curative intent therapy.

\section{Discussion}

\section{Post-operative radiotherapy after EPP}

Radiotherapy has been traditionally used following surgical resection of MPM, with a majority of studies focusing on the safety and efficacy of radiotherapy after EPP. A study on 100 patients with mesothelioma who underwent EPP found that median overall survival was 10.2 months (11). Median overall survival was increased to 14.2 months in the 63 of these patients who also received IMRT, and the 3-year survival of those who received IMRT with epithelioid histology was 28 months. Thirteen percent had local recurrence and $54 \%$ had distant metastases after radiotherapy, implying the efficacy of IMRT in controlling local recurrence but the continued need for systemic therapy. A phase II trial of mesothelioma patients undergoing chemotherapy followed by EPP and possibly radiotherapy found that survival increased from 21.9 to 29.1 months with adjuvant radiotherapy compared to chemotherapy and EPP alone (12).

A later study conducted at MD Anderson on 86 patients who received IMRT after EPP found that recurrence in the ipsilateral hemithorax occurred in only $16 \%$ of patients during the median follow up time of 10.2 months, although $59 \%$ of patients had distant metastases (13). Overall survival was 14.7 months. Patients with epithelioid tumors and negative mediastinal lymph nodes had improved survival compared to others, implying increased utility of radiotherapy in this specific patient population. Almost all patients experienced GI symptoms Grade 2 or higher, and five patients experienced fatal Grade 5 pulmonary toxicity. Of these five, three were toxicity from radiation pneumonitis; reinforcing that risk of lung toxicity must be weighed against the benefits from locoregional control. A later study by Simon et al. analyzed 78 patients undergoing IMRT post or without EPP from 2007-2016; no subject has Grade 3 or high toxicities (14). Patients received a similar dosage as the previous trial; however, authors used a restricted field approach that was shown to have improved target coverage and normal tissues sparing (15). The majority of these patients in this study $(78 \%)$ had the epithelioid subtype.

A phase II trial by Federico et al. studied pre-operative chemotherapy, surgery, and post-operative hemithoracic radiation in 54 patients with MPM (16). Forty-five subjects underwent surgery, with 32 of these subjects receiving radiotherapy, and 22 subjects completing the full treatment course. The median event-free survival was 6.9 months with 
Table 1 Studies on radiation therapy following extrapleural pneumonectomy

\begin{tabular}{lcccccl}
\hline Authors & Year & $\begin{array}{c}\text { Subjects } \\
\text { receiving RT }\end{array}$ & $\begin{array}{c}\text { Radiation } \\
\text { technique }\end{array}$ & $\begin{array}{c}\text { Median } \\
\text { dose }\end{array}$ & Median OS & Toxicity \\
\hline Rice et al. & 2007 & 63 & IMRT & 45 Gy & 14.2 months & Severe respiratory distress: 1 patient \\
Krug et al. & 2009 & 44 & Not specified & 45.9 Gy & 29.1 months & Radiation pneumonitis: 2 patients \\
Gomez et al. & 2013 & 86 & IMRT & & 14.7 months & Grade 5 pulmonary toxicity: 5 patients \\
Simon et al. & 2018 & 27 & IMRT & 54 Gy & 34.9 months (mean) & No Grade 3+ toxicities \\
Federico et al. & 2013 & 32 & Not specified & 50.4 Gy & & $\begin{array}{l}\text { 13\% experienced serious adverse events related } \\
\text { to radiotherapy }\end{array}$ \\
& & & & & & Grade 4+ pneumonitis: 2 patients \\
\hline
\end{tabular}

Overall survival and serious toxicities are reported specifically for patients receiving radiotherapy when provided by the author. RT, radiation therapy; IMRT, intensity modulated radiation therapy; OS, overall survival.

$33.3 \%$ and $24.1 \%$ of patient remaining event-free at 1 and 2 years respectively. The authors concluded the trimodal therapy was feasible with manageable toxicity profile; however, analyzes were limited to overall efficacy outcomes and not stratified by treatment completion and whether subjects received radiotherapy. Authors emphasized that patients should be carefully selected for trimodal therapy.

More recently, the Phase II SAKK 17/04 trial evaluated the effect of radiotherapy after EPP on local relapse-free survival defined as relapse within the ipsilateral hemithorax or death from any cause (17). Patients were initially treated with neoadjuvant cisplatin and pemetrexed prior to EPP. If the patients had a complete macroscopic resection they were randomized to observation or high dose radiotherapy. Two clinical target volumes (CTVs) were defined as field of surgery (including entire hemothorax, thoracotomy channel, and mediastinum if involved/violated during surgery) and a boost which involved areas of highest risk for intrathoracic relapse. These volumes were expanded by $0.5-1 \mathrm{~cm}$ to the planning target volumes (PTV) where radiotherapy was targeted. Three types of dose and fractionation were given depending on available technology and treatment standards of different centers in this multi-institutional trial. This included 45 Gy large volume with a 12.6 Gy boost all in 1.8 Gy fractions; 46 Gy with a 10 Gy boost in 2 Gy per fraction, and 45.5 Gy in 26 fractions with a simultaneous boost to 55.9 Gy. After a median follow-up period of 17 months, the local relapse rate was $24 \%$. Progression-free survival remained low largely due to a high distant relapse rate of $81 \%$, with the majority of this being outside the radiotherapy field. Additionally, while authors concluded that results did not support the routine use of radiation therapy after EPP, the trial had several limitations including lack of statistical power, and experts have continued to recommend radiation therapy in conjunction with EPP despite the results of this trial (18). Table 1 summarizes the above studies and reported median overall survival for patients undergoing EPP and subsequent radiotherapy.

Compared to conventional radiotherapy, IMRT improves target volume coverage at the expense of increasing the radiation dose to the contralateral lung, with an increase in mean lung dose (MLD) and percentage lung volume receiving 13 Gy (V13) and 20 Gy (V20) (7). A study at MD Anderson evaluated the dose dependent pulmonary toxicity after IMRT for MPM in 63 patients. The $V_{20}$ was predictive of pulmonary-related death on multivariate analysis, suggesting that $V_{20}$ should be kept as low as possible (19). Mean lung doses of $>8.5$ Gy were associated with pulmonary-related death on univariate analysis but not multivariate analysis. Based on the results, authors aimed to keep the MLD $<8.5$ Gy and mean $V_{20}<7 \%$. A small retrospective study done at Dana Farber Cancer Institute reviewed the records of 13 MPM patients who received IMRT after EPP and adjuvant chemotherapy, 6 of whom developed fatal pneumonitis (8). The median $V_{20}$, mean lung dose, and $\mathrm{V}_{5}$ (volume of lung receiving 5 Gy or more) were $17.6 \%, 15.2 \mathrm{~Gy}$, and $98.6 \%$ respectively for subjects who developed pneumonitis compared to $10.9 \%, 12.9 \mathrm{~Gy}$, and $90 \%$ respectively for subjects who did not develop pneumonitis; however, none of these three comparisons were statistically significant. Both of these papers indicate the importance reducing the mean lung dose and $\mathrm{V}_{20}$ when using IMRT. Current guidelines from the National Comprehensive Cancer Network recommend that the mean 
Table 2 Studies on radiation therapy following pleurectomy-decortication

\begin{tabular}{lcccccc}
\hline Authors & Year & $\begin{array}{c}\text { Subjects } \\
\text { receiving RT }\end{array}$ & $\begin{array}{c}\text { Radiation } \\
\text { technique }\end{array}$ & Median dose & Median OS & Toxicity \\
\hline Rosenzweig et al. & 2012 & 36 & IMRT & 46.8 Gy & 18 months & Grade 4 + pneumonitis: 2 patients \\
Gupta et al. & 2005 & 123 & EBRT & 42.5 Gy & 13.5 months & Grade 4 + pneumonitis: 2 patients \\
Rimner et al. & 2016 & 27 & IMRT & 46.8 Gy & 23.7 months & No Grade $4+$ toxicities \\
\hline
\end{tabular}

Overall survival and serious toxicities are reported specifically for patients receiving radiotherapy. RT, radiation therapy; IMRT, intensity modulated radiation therapy; EBRT, external beam radiation therapy; OS, overall survival.

lung dose is kept as low as possible, preferably <8.5 Gy (20).

\section{Post-operative radiotherapy after P/D}

Given the demonstrated survival benefit of lung-sparing $\mathrm{P} / \mathrm{D}$ surgery over EPP, this surgery is increasingly preferred to EPP (21). Rosenzweig et al. studied IMRT in 36 patients with MPM, 20 of whom received P/D prior to radiotherapy (22). Patients who received P/ $\mathrm{D}$ and IMRT had a median survival of 26 months from diagnosis; survival rate was $75 \%$ and $53 \%$ at 1 and 2 years respectively. These patients had a local and distant failure rate during the first 12 months of $48 \%$ and $10 \%$ respectively. These survival rates were an improvement from a previous study at the same institution on patients who received $\mathrm{P} / \mathrm{D}$ and adjuvant conventional radiotherapy, with a median survival of 13.5 months and overall 2-year survival of $23 \%$ (23).

In the IMPRINT phase II trial, authors evaluated the safety of hemithoracic intensity-modulated pleural radiation therapy (IMPRINT) in patients who had undergone pleurectomy-decortication (P/D) and chemotherapy (24). Given the lung-sparing nature of the P/ $\mathrm{D}$, radiation techniques must spare both the ipsilateral and contralateral lung tissue. 27 patients were evaluated. No grade 4 or 5 toxicities were seen; eight patients developed grade 2 or 3 radiation pneumonitis that improved with steroid administration. Five patients experienced Grade 3 fatigue. Authors concluded that IMRT in patients with $\mathrm{P} / \mathrm{D}$ was safe, with an acceptable rate of radiation pneumonitis. A phase III trial to determine overall survival and local-failure-free survival between a treatment arm that receives $\mathrm{P} / \mathrm{D}$ and chemotherapy and a treatment arm that receives $\mathrm{P} / \mathrm{D}$, chemotherapy, and IMRT is currently underway (Clinicaltrials.gov NCT04158141) (25). Table 2 summarizes the aforementioned studies on radiotherapy after P/D.
A recent Phase III trial investigated whether radical hemithoracic radiation (RHR) therapy would improve overall survival compared with palliative radiation $(\mathrm{PR})$ therapy in subjects with MPM who underwent lungsparing surgery (26). A total of 108 patients who received either $\mathrm{P} / \mathrm{D}$, extended $\mathrm{P} / \mathrm{D}$, or partial pleurectomy were randomized to receive either RHR or PR. Authors found a statistically significant benefit of RHR, with the 2 year overall survival rate $58 \%$ in the RHR compared to $28 \%$ in the $\mathrm{PR}$ arm $(\mathrm{P}=0.031)$. Notably, toxicity was higher in the RHR arm, with $20 \%$ of patients experiencing Grade 3 toxicity or greater, compared with no patients experiencing Grade 3 toxicity or greater in the PR arm. Sixteen percent of patients in the RHR arm experienced Grade 2 or greater pneumonitis, including one possibly fatal event. While RHR is promising for increased survival of patients with MPM who receive lung-sparing surgery, patients must be carefully selected given the non-negligible side effects of increased radiation doses. A consensus expert opinion from the National Cancer Institute, International Association for the Study of Lung Cancer Research, and Mesothelioma Applied Research Foundation recommends, based on the current literature, that in patients with two intact lungs, the mean lung dose should be kept to 20.5 Gy or less, the total lung $\mathrm{V}_{20}$ (volume receiving $20 \mathrm{~Gy}$ or more) to $37 \%$ or less, the ipsilateral lung $\mathrm{V}_{40}$ (volume receiving 40 Gy or more) to $67 \%$ or less, and the contralateral lung dose to $<8$ Gy (18). Clinical practice guidelines from the American Society of Clinical Oncology recommend that IMRT following lung-sparing surgery should be performed only at highly experienced centers, preferably in the context of a clinical trial, given the toxicity concerns (27).

\section{Pre-operative radiotherapy}

De Perrot et al. developed a protocol named surgery for mesothelioma after radiation therapy (SMART), which 
was a reversal of the traditional protocol of surgery followed by radiation (6). Intensity-modulated radiation therapy (IMRT) was administered to 62 patients. EPP was performed within 2 weeks of the last dose of radiotherapy. Median overall survival was 51 months in epithelioid subtype versus 10 months in biphasic subtype. A total of 30 patients developed tumor recurrence, most commonly seen in the contralateral chest $(n=9)$ or abdomen $(n=8)$; 8 patients developed recurrence in the ipsilateral chest. Of those 8 patients, the majority of patients $(n=5)$ had the biphasic subtype. An important consideration with this approach is that patients must be able to proceed to surgery following radiation treatment, which may limit the number of candidates given the possible toxicity of the radiotherapy. More research is warranted on pre-operative radiotherapy for MPM.

\section{Role of radiation in procedure tract metastases}

The SMART trial (not to be confused with the aforementioned surgery for mesothelioma after radiation therapy trial) was aimed to determine whether immediate radiotherapy given within 42 days of pleural intervention reduced the incidence of procedure-tract metastases (PTM) versus delayed radiotherapy in subjects with histologically proven MPM (28). A total of 203 patients were randomized to receive immediate radiotherapy $(\mathrm{n}=102)$ or deferred radiotherapy $(\mathrm{n}=101)$ after large-bore pleural interventions. No significant difference was found overall in incidence of PTM, analgesia use, survival, or quality of life. However, when authors performed a subgroup analysis on subjects with epithelioid tumors, prophylactic radiotherapy suggested some benefit over delayed radiotherapy with $6 / 71(8 \%)$ of subjects and 15/72 (21\%) developing PTM respectively $(\mathrm{P}=0.06)$. The results from this trial aligned those from a meta-analysis of five randomized controlled trials, which concluded that prophylactic radiotherapy did not reduce procedure site recurrence (29). Consensus recommendations from a multidisciplinary team of mesothelioma expects do not recommend radiation to prevent PTMs, with the exception of patients with histologically confirmed positive procedure tracts (18). More research is warranted on specifically for the population with epithelioid tumors to determine if there is any benefit.

\section{Radiotherapy with non-operative management}

The overall survival benefit of MPM patients after undergoing a major surgical resection such as EPP or P/D depends largely on patient factors. Patients with sarcomatoid or biphasic histology, nodal disease, elevated platelets, and advanced age have been shown to be poor surgical candidates (30). Unresectable disease is typically managed with chemotherapy alone (31). For nonresectable tumors or patients with poor surgical prognostic factors, limited studies have demonstrated that radiotherapy may be used as curative intent therapy. A study by Jenkins et al. found that in 54 MPM patients receiving palliative radiotherapy, there was a response rate of $43 \%$, with 22 patients achieving a partial response and 1 patient achieving a complete response (4). Response rates were similar to phase II studies of chemotherapy for MPM (32). Survival correlated favorably with non-sarcomatous histological subtype. A retrospective study using 23,414 patients with mesothelioma in the National Cancer Data Base (NCDB) found that compared with subjects who did not undergo surgery or definitive radiotherapy, subjects who received both surgery and radiotherapy had the largest decrease in mortality with a hazard ratio (HR) of 0.60 (95\% CI: 0.53-0.69). Subjects who received definitive radiotherapy alone had decreased mortality with a $\mathrm{HR}=0.74$ (95\% CI: 0.62-0.88), and subjects who received surgery alone had similarly decreased mortality with a HR=0.75 (95\% CI: 0.71-0.80) (33). These findings suggest that definitive radiotherapy is a useful option both in combination with surgery and for patients with unresectable disease.

Lastly, radiotherapy has been widely used to alleviate pain in MPM and may be of benefit for symptom relief in patients with nonresectable tumors. A systemic review article published in 2014 reported that there is no high quality evidence exists to support the routine use of radiotherapy for pain relief (34). Following this, this SYSTEMS Phase II trial assessed the role of radiotherapy in pain management for MPM (35). Fourteen (35\%) of patients had a clinically significant improvement in pain 5 weeks post radiotherapy, with $38 \%, 40 \%$, and $66.6 \%$ of epithelioid, sarcomatoid, and mixed histology patients responding, respectively. Importantly, results demonstrated pain relief may be achieved regardless of tumor histological subtype. While studies are limited, demonstrated tumor response and symptom relief shows a promising role for radiotherapy in the management of nonresectable MPM. Additionally, recently published clinical guidelines recommend offering palliative radiation therapy in patients with symptomatic disease (27). 


\section{Tumor beterogeneity}

As detailed in the above sections, more favorable results were achieved with radiotherapy used for the epithelioid subtype of MPM as opposed to the sarcomatoid subtype. However, recent research has emphasized the intratumor heterogeneity of certain malignant neoplasms. This intratumor heterogeneity may allow some tumor cells to survive during targeted therapy, contributing to acquired resistance and relapse of the neoplasm (36). MPM has been shown to have both inter-tumor heterogeneity between patients and intra-tumor heterogeneity within a tumor sample of a given patient (37). A study by Kiyotani et al. found heterogeneity in both the genomic landscape and immune microenvironment of MPM (38). Nonsynonymous mutations and gene expression profiles differed among individual tumors as well as different tumor sites in an individual patient. Authors concluded that a single tumor-biopsy specimen may not be adequate to characterize the tumor nature; however, obtaining multiple biopsy specimens remains challenging. Blum et al. described MPM heterogeneity using a bioinformatics method called WISP, allowing the proportion of epithelioid and sarcomatoid morphologies of a given tumor to be taken into account. Authors compared survival for patients with $\geq 22 \%$ of S-score (percentage of sarcomatoid components) to those with $<22 \%$ of S-score and found a hazard ratio of 6.28 $(\mathrm{P}=0.001)$ (39), with a difference in median overall survival of greater than 10 months between the groups. Research in the area of tumor heterogeneity in MPM is relatively new but an important consideration moving forward given the prognostic factors of different tumor subtypes and the treatment decisions made based on patient prognosis, particularly as it relates to our discussion of radiotherapy.

\section{Conclusions}

Unfortunately, the overall prognosis for mesothelioma remains poor. Radiation therapy has been shown to be effective in reducing local recurrence and appears to be especially well-suited for epithelioid cell types. Additionally, radiation therapy prior to surgery has shown benefit for certain patients, especially those with early-stage epithelioid tumors, although current evidence is limited. While adverse effects from radiation, including radiation pneumonitis are not insignificant, recent technical advances have improved local control and decreased the radiation dosage to the contralateral lung. Radiotherapy appears to be safe with tolerable side effects in conjunction with either EPP or P/D, although patients should be carefully selected.

\section{Acknowledgments}

Funding: None.

\section{Footnote}

Provenance and Peer Review: This article was commissioned by the Guest Editors (Abbas E. Abbas and Stacey Su) for the series "Mesothelioma" published in AME Surgical fournal. The article has undergone external peer review.

Reporting Checklist: The authors have completed the Narrative Review reporting checklist. Available at https:// asj.amegroups.com/article/view/10.21037/asj-21-26/rc

Peer Review File: Available at https://asj.amegroups.com/ article/view/10.21037/asj-21-26/prf

Conflicts of Interest: Both authors have completed the ICMJE uniform disclosure form (available at https://asj.amegroups. com/article/view/10.21037/asj-21-26/coif). The series "Mesothelioma" was commissioned by the editorial office without any funding or sponsorship. The authors have no other conflicts of interest to declare.

Ethical Statement: The authors are accountable for all aspects of the work in ensuring that questions related to the accuracy or integrity of any part of the work are appropriately investigated and resolved.

Open Access Statement: This is an Open Access article distributed in accordance with the Creative Commons Attribution-NonCommercial-NoDerivs 4.0 International License (CC BY-NC-ND 4.0), which permits the noncommercial replication and distribution of the article with the strict proviso that no changes or edits are made and the original work is properly cited (including links to both the formal publication through the relevant DOI and the license). See: https://creativecommons.org/licenses/by-nc-nd/4.0/.

\section{References}

1. Mutti L, Peikert T, Robinson BWS, et al. Scientific Advances and New Frontiers in Mesothelioma Therapeutics. J Thorac Oncol 2018;13:1269-83. 
2. Zucali PA, De Vincenzo F, Simonelli M, et al. Future developments in the management of malignant pleural mesothelioma. Expert Rev Anticancer Ther 2009;9:453-67.

3. Cramer G, Simone CB 2nd, Busch TM, et al. Adjuvant, neoadjuvant, and definitive radiation therapy for malignant pleural mesothelioma. J Thorac Dis 2018;10:S2565-73.

4. Jenkins P, Milliner R, Salmon C. Re-evaluating the role of palliative radiotherapy in malignant pleural mesothelioma. Eur J Cancer 2011;47:2143-9.

5. Pass HI, Kranda K, Temeck BK, et al. Surgically debulked malignant pleural mesothelioma: results and prognostic factors. Ann Surg Oncol 1997;4:215-22.

6. de Perrot M, Wu L, Wu M, et al. Radiotherapy for the treatment of malignant pleural mesothelioma. Lancet Oncol 2017;18:e532-42.

7. Krayenbuehl J, Oertel S, Davis JB, et al. Combined photon and electron three-dimensional conformal versus intensity-modulated radiotherapy with integrated boost for adjuvant treatment of malignant pleural mesothelioma after pleuropneumonectomy. Int J Radiat Oncol Biol Phys 2007;69:1593-9.

8. Allen AM, Czerminska M, Jänne PA, et al. Fatal pneumonitis associated with intensity-modulated radiation therapy for mesothelioma. Int J Radiat Oncol Biol Phys 2006;65:640-5.

9. Pan HY, Jiang S, Sutton J, et al. Early experience with intensity modulated proton therapy for lungintact mesothelioma: A case series. Pract Radiat Oncol 2015;5:e345-53.

10. Hanna GG, John T, Ball DL. Controversies in the role of radiotherapy in pleural mesothelioma. Transl Lung Cancer Res 2021;10:2079-87.

11. Rice DC, Stevens CW, Correa AM, et al. Outcomes after extrapleural pneumonectomy and intensity-modulated radiation therapy for malignant pleural mesothelioma. Ann Thorac Surg 2007;84:1685-92; discussion 1692-3.

12. Krug LM, Pass HI, Rusch VW, et al. Multicenter phase II trial of neoadjuvant pemetrexed plus cisplatin followed by extrapleural pneumonectomy and radiation for malignant pleural mesothelioma. J Clin Oncol 2009;27:3007-13.

13. Gomez DR, Hong DS, Allen PK, et al. Patterns of failure, toxicity, and survival after extrapleural pneumonectomy and hemithoracic intensity-modulated radiation therapy for malignant pleural mesothelioma. J Thorac Oncol 2013;8:238-45.

14. Simon M, Shochat T, Peled N, et al. Intensity-modulated radiotherapy is a safe and effective treatment for localized malignant pleural mesothelioma. Thorac Cancer
2018;9:1470-5.

15. Allen AM, Schofield D, Hacker F, et al. Restricted field IMRT dramatically enhances IMRT planning for mesothelioma. Int J Radiat Oncol Biol Phys 2007;69:1587-92.

16. Federico R, Adolfo F, Giuseppe M, et al. Phase II trial of neoadjuvant pemetrexed plus cisplatin followed by surgery and radiation in the treatment of pleural mesothelioma. BMC Cancer 2013;13:22.

17. Stahel RA, Riesterer O, Xyrafas A, et al. Neoadjuvant chemotherapy and extrapleural pneumonectomy of malignant pleural mesothelioma with or without hemithoracic radiotherapy (SAKK 17/04): a randomised, international, multicentre phase 2 trial. Lancet Oncol 2015;16:1651-8.

18. Gomez DR, Rimner A, Simone CB 2nd, et al. The Use of Radiation Therapy for the Treatment of Malignant Pleural Mesothelioma: Expert Opinion from the National Cancer Institute Thoracic Malignancy Steering Committee, International Association for the Study of Lung Cancer, and Mesothelioma Applied Research Foundation. J Thorac Oncol 2019;14:1172-83.

19. Rice DC, Smythe WR, Liao Z, et al. Dose-dependent pulmonary toxicity after postoperative intensity-modulated radiotherapy for malignant pleural mesothelioma. Int J Radiat Oncol Biol Phys 2007;69:350-7.

20. Ettinger DS, Wood DE, Akerley W, et al. NCCN Guidelines Insights: Malignant Pleural Mesothelioma, Version 3.2016. J Natl Compr Canc Netw 2016;14:825-36.

21. Flores RM, Pass HI, Seshan VE, et al. Extrapleural pneumonectomy versus pleurectomy/decortication in the surgical management of malignant pleural mesothelioma: results in 663 patients. J Thorac Cardiovasc Surg 2008;135:620-6, 626.e1-3.

22. Rosenzweig KE, Zauderer MG, Laser B, et al. Pleural intensity-modulated radiotherapy for malignant pleural mesothelioma. Int J Radiat Oncol Biol Phys 2012;83:1278-83.

23. Gupta V, Mychalczak B, Krug L, et al. Hemithoracic radiation therapy after pleurectomy/decortication for malignant pleural mesothelioma. Int J Radiat Oncol Biol Phys 2005;63:1045-52.

24. Rimner A, Zauderer MG, Gomez DR, et al. Phase II Study of Hemithoracic Intensity-Modulated Pleural Radiation Therapy (IMPRINT) As Part of Lung-Sparing Multimodality Therapy in Patients With Malignant Pleural Mesothelioma. J Clin Oncol 2016;34:2761-8.

25. Testing the Addition of Targeted Radiation Therapy 
to Surgery and the Usual Chemotherapy Treatment (Pemetrexed and Cisplatin [or Carboplatin]) for Stage I-IIIA Malignant Pleural Mesothelioma. ClinicalTrials. gov Identifier: NCT04158141. Available from: https:// clinicaltrials.gov/ct2/show/NCT04158141

26. Trovo M, Relevant A, Polesel J, et al. Radical Hemithoracic Radiotherapy Versus Palliative Radiotherapy in Nonmetastatic Malignant Pleural Mesothelioma: Results from a Phase 3 Randomized Clinical Trial. Int J Radiat Oncol Biol Phys 2021;109:1368-76.

27. Kindler HL, Ismaila N, Armato SG 3rd, et al. Treatment of Malignant Pleural Mesothelioma: American Society of Clinical Oncology Clinical Practice Guideline. J Clin Oncol 2018;36:1343-73.

28. Clive AO, Taylor H, Dobson L, et al. Prophylactic radiotherapy for the prevention of procedure-tract metastases after surgical and large-bore pleural procedures in malignant pleural mesothelioma (SMART): a multicentre, open-label, phase 3, randomised controlled trial. Lancet Oncol 2016;17:1094-104.

29. Bergamin S, Tio M, Stevens MJ. Prophylactic procedure tract radiotherapy for malignant pleural mesothelioma: A systematic review and meta-analysis. Clin Transl Radiat Oncol 2018;13:38-43.

30. Woodard GA, Jablons DM. Surgery for pleural mesothelioma, when it is indicated and why: arguments against surgery for malignant pleural mesothelioma. Transl Lung Cancer Res 2020;9:S86-91.

31. Kelly RJ, Sharon E, Hassan R. Chemotherapy and targeted therapies for unresectable malignant mesothelioma. Lung

doi: 10.21037/asj-21-26

Cite this article as: Saldanha G, Kumar S. Role of radiation treatment in multimodality treatment of malignant pleural mesothelioma: a narrative review. AME Surg J 2021;1:23.
Cancer 2011;73:256-63.

32. van Meerbeeck JP, Baas P, Debruyne C, et al. A Phase II study of gemcitabine in patients with malignant pleural mesothelioma. European Organization for Research and Treatment of Cancer Lung Cancer Cooperative Group. Cancer 1999;85:2577-82.

33. Ohri N, Taioli E, Ehsani M, et al. Definitive Radiation Therapy Is Associated With Improved Survival in NonMetastatic Malignant Pleural Mesothelioma. Int J Radiat Oncol 2016;96:S132-3.

34. Macleod N, Price A, O'Rourke N, et al. Radiotherapy for the treatment of pain in malignant pleural mesothelioma: a systematic review. Lung Cancer 2014;83:133-8.

35. MacLeod N, Chalmers A, O'Rourke N, et al. Is Radiotherapy Useful for Treating Pain in Mesothelioma?: A Phase II Trial. J Thorac Oncol 2015;10:944-50.

36. Marusyk A, Janiszewska M, Polyak K. Intratumor Heterogeneity: The Rosetta Stone of Therapy Resistance. Cancer Cell 2020;37:471-84.

37. Oehl K, Vrugt B, Opitz I, et al. Heterogeneity in Malignant Pleural Mesothelioma. Int J Mol Sci 2018;19:1603.

38. Kiyotani K, Park JH, Inoue H, et al. Integrated analysis of somatic mutations and immune microenvironment in malignant pleural mesothelioma. Oncoimmunology 2017;6:e1278330.

39. Blum Y, Meiller C, Quetel L, et al. Dissecting heterogeneity in malignant pleural mesothelioma through histo-molecular gradients for clinical applications. Nat Commun 2019;10:1333. 